\title{
Prognostic factors and skeletal-related events in patients with bone metastasis from gastric cancer
}

\author{
YOSHINORI IMURA ${ }^{1}$, DAISUKE TATEIWA ${ }^{2}$, NAOTOSHI SUGIMOTO ${ }^{3}$, AKITOMO INOUE ${ }^{1}$, \\ TORU WAKAMATSU ${ }^{1}$, HIDETATSU OUTANI $^{2}$, TAKAAKI TANAKA $^{1}$, HIRONARI TAMIYA $^{1}$, TOSHINARI YAGI $^{3}$, \\ NORIFUMI NAKA ${ }^{1}$, SUMIYO OKAWA $^{4}$, TAKAHIRO TABUCHI ${ }^{4}$ and SATOSHI TAKENAKA ${ }^{1}$ \\ ${ }^{1}$ Musculoskeletal Oncology Service, Osaka International Cancer Institute, Osaka 541-8567; \\ ${ }^{2}$ Department of Orthopedic Surgery, Osaka University Graduate School of Medicine, Suita, Osaka 565-0871; \\ ${ }^{3}$ Department of Clinical Oncology; ${ }^{4}$ Cancer Control Center, Osaka International Cancer Institute, Osaka 541-8567, Japan
}

Received November 28, 2019; Accepted June 16, 2020

DOI: $10.3892 / \mathrm{mco} .2020 .2101$

\begin{abstract}
The number of studies on bone metastasis (BM) from gastric cancer $(\mathrm{GC})$ is currently limited. Therefore, the aim of the present study was to investigate the characteristics, skeletal-related events (SREs) and prognosis of GC in patients with BMs. Data from 60 patients with BMs from GC were retrospectively retrieved and patient-, tumor- and BM-related characteristics were analyzed. Kaplan-Meier survival curves were analyzed using the univariate log-rank test. Multivariate analyses were conducted using the Cox proportional hazards model. The median patient age was 63.5 years (range, 26-83 years). Visceral or brain metastases were observed at BM diagnosis in $61.7 \%$ of the patients. Multiple BMs were detected in $83.3 \%$ and SREs occurred in $76.7 \%$ of the patients. The median overall survival (OS) after BM diagnosis and SRE occurrence was 9 months (range, 0-43 months) and 5 months (range, 0-36 months), respectively. On multivariate analysis, poor Eastern Cooperative Oncology Group performance status $(\mathrm{P}=0.030)$, the administration of chemotherapy prior to $\mathrm{BM}$ diagnosis $(\mathrm{P}<0.001)$ and no chemotherapy after $\mathrm{BM}$ diagnosis $(\mathrm{P}=0.002)$ were significant prognostic factors for unfavorable OS, whereas the non-use of bone-modifying agents (BMAs) was the only independent prognostic factor for poor SRE-free survival (SRS; $\mathrm{P}=0.022$ ). Among patients without SREs at BM diagnosis, the median SRS duration was 7 months (range, 0-43 months). In conclusion, chemotherapy may confer a survival benefit in GC patients with BMs. In addition, the prognosis for $\mathrm{GC}$ patients with BMs presenting with SREs is poor, but treatment with BMAs may prevent or delay the development of SREs.
\end{abstract}

Correspondence to: $\mathrm{Dr}$ Yoshinori Imura, Musculoskeletal Oncology Service, Osaka International Cancer Institute, 3-1-69 Otemae, Chuo-ku, Osaka 541-8567, Japan

E-mail: y.imura@hotmail.co.jp

Key words: bone metastasis, gastric cancer, skeletal-related event

\section{Introduction}

Gastric cancer (GC) is the fourth most common malignancy and the second leading cause of cancer-related mortality worldwide (1). Following surgery or late during the clinical course, GC frequently spreads to the regional lymph nodes, liver, peritoneum and lungs (2), but rarely disseminates to the bones. Thus, the incidence of bone metastasis (BM) from GC is only $0.9-10.5 \%$ (3-7), although the reported frequency of BM in GC patients is $13.4 \%$ in autopsy series and increases up to $45.3 \%$ in BM screening studies $(8,9)$. Therefore, the incidence of asymptomatic BM may be underestimated, and the rate of BM in clinical cases may be markedly higher compared with the reported incidence.

The bone is a frequent metastatic site of breast, prostate and lung cancers, and the presence of BM typically indicates a poor prognosis (10-12). The incidence and prevalence of BM have increased proportionally with the aging population. Skeletal-related events (SREs), including pathological fractures, spinal cord compression and hypercalcemia, are the result of BM, may cause reduced physical function and quality of life (QoL), and require treatment with radiotherapy or surgery (13). A multidisciplinary approach using modalities such as radiotherapy, surgery and various medical treatments, including chemotherapy, hormone therapy and bone-modifying agents (BMA), is therefore warranted for patients with BM (10).

The presence of BM in metastatic GC has been reported as an independent poor prognostic factor, and GC patients with BMs exhibited the poorest median survival time compared with patients with metastases to other sites, including the chest, liver, or abdomen (14). However, to date, there have been only few studies examining the clinical presentation and prognosis of BM from GC.

The aim of the present study was to retrospectively investigate the clinicopathological characteristics, treatment outcomes, prognostic factors and SREs in GC patients with $\mathrm{BM}$ who underwent treatment at our institution.

\section{Patients and methods}

Study design and patients. The medical records of $60 \mathrm{GC}$ patients with BM who were treated at the Osaka International 
Cancer Institute between January 2005 and December 2017 were anonymized and retrospectively reviewed following BM diagnosis by physicians, radiologists and orthopedic surgeons. The diagnosis of BM was based on clinical symptoms and signs, as well as radiographic imaging studies, such as X-ray, computed tomography (CT), magnetic resonance imaging (MRI), bone scintigraphy, and/or fluorodeoxyglucose-positron emission tomography (FDG-PET)/CT. Pathological fractures, spinal cord compression and hypercalcemia were defined as SREs, as were radiotherapy and orthopedic surgery that were performed for BM. The protocol of the present study was approved by the Institutional Review Board of the Osaka International Cancer Institute.

Data collection. The data collected for the present study were as follows: Patient-related characteristics, including age, sex and Eastern Cooperative Oncology Group performance status (ECOG PS) score; tumor-related characteristics, including histology, differentiation, stage, resection of primary site, visceral or brain metastasis, and levels of serum carcinoembryonic antigen (CEA), carbohydrate antigen 19-9 (CA19-9), C-reactive protein (CRP), lactate dehydrogenase (LDH), albumin and alkaline phosphatase (ALP); BM-related characteristics, including clinical symptoms, the presence of BM at the initial diagnosis of GC, number, location, type, treatment received (chemotherapy before and after the BM diagnosis, and BMA) and SREs; follow-up period; and outcome at last follow-up.

Follow-up and outcomes. The median follow-up period for all patients was 5 months (range, 0-43 months). Overall survival (OS) was defined as the time from the date of diagnosis of BM to the date of death from any cause or the date of the last follow-up visit. SRE-free survival (SRS) was defined as the time from the date of BM diagnosis to the date of the first SRE occurrence or the last follow-up visit.

Statistical analysis. The Kaplan-Meier method was used to calculate OS and SRS. The impact of prognostic factors on OS and SRS was first assessed using the log-rank test in univariate analysis, and multivariate analysis was then performed using the Cox proportional hazard model. The significant variables in the univariate model except for intervening variables were used to build the multivariate model of survival. $\mathrm{P}<0.05$ was considered to indicate statistically significant differences. Statistical analyses were performed using EZR software version 1.35 (Saitama Medical Center, Jichi Medical University, Saitama, Japan), which is a graphical user interface for R (The R Foundation for Statistical Computing, Vienna, Austria).

\section{Results}

Patient, tumor and BM-related characteristics. The patient, tumor and BM-related characteristics of the 60 cases are summarized in Table I. A total of 33 patients (55\%) were male. The median age at diagnosis of BM was 63.5 years (range, 26-83 years). The ECOG PS score was 0-2 in 42 patients (70\%) and 3-4 in 18 patients (30\%). Tumor histology of the primary lesion was adenocarcinoma in 56 patients (93.3\%) and scirrhous carcinoma in 4 patients (6.7\%). A total of 14 patients (23.3\%) had well- to moderately differentiated tumors, and 46 (76.7\%) had poorly differentiated tumors. A total of 4 patients $(6.7 \%)$ had stage I, $6(10 \%)$ had stage II, 11 (18.3\%) had stage III, 36 $(60 \%)$ had stage IV, and $3(5 \%)$ had unknown-stage disease at initial diagnosis. In addition, 26 patients (43.3\%) underwent surgery for the primary tumor. Visceral or brain metastasis coexisted with BM at the time of BM diagnosis in 37 patients (61.7\%). The proportion of patients with elevated levels of serum CEA ( $>5 \mathrm{ng} / \mathrm{ml})$, CA19-9 ( $>37 \mathrm{U} / \mathrm{ml}), \mathrm{CRP}(>0.3 \mathrm{mg} / \mathrm{dl}), \mathrm{LDH}$ $(>250 \mathrm{U} / \mathrm{l})$ and ALP $(>350 \mathrm{U} / \mathrm{l})$ at the time of BM diagnosis were $62.7,60,44.1,38.3$ and $63.3 \%$, respectively. Decreased serum albumin level $(\leq 3.7 \mathrm{~g} / \mathrm{dl})$ was detected in $49.2 \%$ of the patients.

BM diagnosis was confirmed with CT, MRI, bone scintigraphy and FDG-PET/CT in 32, 36, 20 and 25 patients, respectively. Clinical symptoms, such as pain and paralysis, were present in 34 patients (56.7\%) at diagnosis of BM, whereas 26 patients $(43.3 \%)$ were asymptomatic. A total of 19 patients (31.7\%) had already developed BM at GC diagnosis. Among the remaining 41 patients (68.3\%) who developed BM after the diagnosis of $\mathrm{GC}$, the median interval from $\mathrm{GC}$ diagnosis to detection of BM was 15 months (range, 1-126 months). A solitary BM was found in 10 patients (16.7\%) and multiple BMs were detected in 50 patients $(83.3 \%)$. The spine was the most common site for BM (49 patients, $81.7 \%$ ), followed by the pelvis (37 patients, 61.7\%), ribs (24 patients, $40 \%$ ) and sternum (15 patients, $25 \%)$. The lesions were osteoblastic in 15 patients (25\%), osteolytic in $24(40 \%)$, mixed in $15(25 \%)$, and intertrabecular in 6 patients $(10 \%)$. Chemotherapy was administered before the diagnosis of BM in 32 patients (53.3\%), and 46 patients (76.7\%) received palliative chemotherapy after BM diagnosis. BMA, such as zoledronic acid and denosumab, were administered after diagnosis of BM to 34 patients $(56.7 \%)$.

SREs. A total of 46 patients $(76.7 \%)$ experienced SREs. The most frequent SREs were radiotherapy (44 patients, 73.3\%) followed by spinal cord compression (17 patients, 28.3\%), pathological fractures (13 patients, 21.7\%), orthopedic surgery (6 patients, 10\%) and hypercalcemia (4 patients, 6.7\%). A total of 23 patients (38.3\%) presented with SREs at the time of BM diagnosis, and the remaining 23 patients (38.3\%) developed SREs during follow-up.

Predictive factors of OS. Among the $60 \mathrm{GC}$ patients with $\mathrm{BMs}$, the OS rates after the diagnosis of BM were $62.3 \%$ (6 months), $44.5 \%$ (1 year), and $12.7 \%$ ( 2 years). The median OS duration after the diagnosis of BM was 9 months (range, 0-43 months). A total of 46 patients who experienced SREs had a median OS duration of 5 months (range, 0-36 months) after the diagnosis of SREs.

On univariate analyses, ECOG PS score $(\mathrm{P}<0.001)$, visceral or brain metastasis $(\mathrm{P}=0.009)$, serum levels of $\mathrm{CRP}(\mathrm{P}=0.041)$, LDH $(\mathrm{P}=0.005)$ and albumin $(\mathrm{P}=0.009)$, patients undergoing chemotherapy prior to $(\mathrm{P}=0.011)$ and after $(\mathrm{P}<0.001)$ the diagnosis of $\mathrm{BM}$ and $\mathrm{SRE}$ at $\mathrm{BM}$ diagnosis $(\mathrm{P}<0.001)$ were significant prognostic factors for OS after the diagnosis of $\mathrm{BM}$ (Table I, Fig. 1A-H). Multivariate analyses revealed that ECOG PS score $>2$ [hazard ratio $(\mathrm{HR})=3.165 ; 95 \%$ confidence interval (CI): $1.120-8.945 ; \mathrm{P}=0.030$ ], undergoing chemotherapy prior to 
Table I. Patient-, tumor- and BM-related characteristics and univariate analysis of prognostic factors for OS $(n=60)$.

\begin{tabular}{lccc}
\hline Factors & $\mathrm{N}(\%)$ & $\begin{array}{c}\text { Median OS } \\
\text { (months) }\end{array}$ & P-value \\
\hline
\end{tabular}

Age (years)
$\leq 60$
$>60$

$>60$

ECOG PS score

$0-2$
$3-4$
Resection of primary site

Present

Visceral or brain

metastasis

Present
Absent
CEA $(n g / m l)$
$\leq 5$
$>5$
N/A

CA19-9 (U/ml)

$\leq 37$
$>37$

CRP (mg/dl)

$\leq 0.3$
$>0.3$
N/A
LDH $(\mathrm{U} / \mathrm{l})$
$\leq 250$
$>250$

Albumin (g/dl)

$$
\begin{aligned}
& \leq 3.7 \\
& >3.7 \\
& \text { N/A }
\end{aligned}
$$

$\operatorname{ALP}(\mathrm{U} / \mathrm{l})$

$$
\begin{aligned}
& \leq 350 \\
& >350
\end{aligned}
$$

34 (56.7)

10

8

42 (70)

12

18 (30)

3

$26(43.3) \quad 8$

$34(56.7)$

11

0.426

$<0.001$

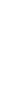

0.009

37 (61.7) $\quad 8$

$23(38.3) \quad 14$

$22(37.3) \quad 12$

$37(62.7) \quad 9$

1

$24(40) \quad 10$

$36(60) \quad 9$

$33(55.9) \quad 12$

$26(44.1) \quad 8$

1

37 (61.7)

23 (38.3)

12

5

$29(49.2) \quad 5$

$30(50.8) \quad 16$

1

$22(36.7) \quad 9$

$38(63.3) \quad 10$

$\mathrm{BM}$ at GC diagnosis

Present

$19(31.7) \quad 12$

Absent

Number of BM

Solitary

Multiple

Chemotherapy before

BM diagnosis

Present

$32(53.3)$

28 (46.7)

5

Absent

Chemotherapy after

BM diagnosis

$\begin{array}{lrr}\text { Present } & 46(76.7) & 12 \\ \text { Absent } & 14(23.3) & 2\end{array}$

0.341

0.329

0.009
Table I. Continued.

\begin{tabular}{lccr}
\hline Factors & $\mathrm{N}(\%)$ & $\begin{array}{c}\text { Median OS } \\
\text { (months) }\end{array}$ & P-value \\
\hline Use of BMA & & & 0.152 \\
Present & $34(56.7)$ & 11 & \\
Absent & $26(43.3)$ & 5 & $<0.001$ \\
SRE at BM diagnosis & & & \\
Present & $23(38.3)$ & 4 & \\
Absent & $37(61.7)$ & 12 & \\
\hline
\end{tabular}

BM, bone metastasis; OS, overall survival; ECOG PS, Eastern Cooperative Oncology Group performance status; CEA, carcinoembryonic antigen; CA19-9, carbohydrate antigen 19-9; CRP, C-reactive protein; LDH, lactate dehydrogenase; ALP, alkaline phosphatase; GC, gastric cancer; BMA, bone-modifying agent; SRE, skeletal-related event; N/A, not available.

BM diagnosis $(\mathrm{HR}=3.802 ; 95 \% \mathrm{CI}: 1.812-7.976 ; \mathrm{P}<0.001)$, and lack of chemotherapy after BM diagnosis $(\mathrm{HR}=5.897 ; 95 \% \mathrm{CI}$ : 1.926-18.050; $\mathrm{P}=0.002$ ) were independently correlated with a shorter OS after the occurrence of BM (Table II).

Predictive factors of SRS. Among the 37 patients without SREs at BM diagnosis, the median SRS duration was 7 months (range, 0-43 months). On univariate analyses, BM at $\mathrm{GC}$ diagnosis $(\mathrm{P}=0.049)$ and the use of BMA $(\mathrm{P}=0.008)$ were significant prognostic factors for SRS (Table III, Fig. 2A and B). Multivariate analyses revealed that the non-use of BMA (HR=2.868; 95\% CI: 1.163-7.076; $\mathrm{P}=0.022)$ was the only independent significant prognostic factor for unfavorable SRS (Table IV).

\section{Discussion}

$\mathrm{BM}$ results in disruption of the normal bone homeostasis, which is a dynamic process involving osteoclast-mediated osteolysis and osteoblast-mediated osteogenesis. This

0.574 frequently decreases bone integrity and causes severe bone pain, an increased risk of fracture, and the release of minerals from the bone matrix, resulting in hypercalcemia $(13,15)$.

0.380 Complications associated with BM include SREs, such as radiotherapy or surgery of the bone, pathological fractures, spinal cord compression and hypercalcemia, which result in morbidity, deterioration of the QoL and poor prognosis.

0.130 Therefore, early diagnosis and appropriate treatment of BM are crucial for preventing the development of SREs and improving patient survival.

0.011 Early detection of BM relies on its clinical characteristics. There were clinical symptoms in $56.7 \%$ of the patients at diagnosis of BM. CT scan is the most commonly used imaging technique for the surveillance of GC patients. Although MRI was not routinely used for patient follow-up monitoring, MRI $<0.001$ was the technique mostly used for diagnosing BM in the present study. Our study demonstrated that skeletal metastatic lesions arising from GC were frequently found at multiple locations $(83.3 \%)$, were metachronous $(68.3 \%)$, coexisted with 

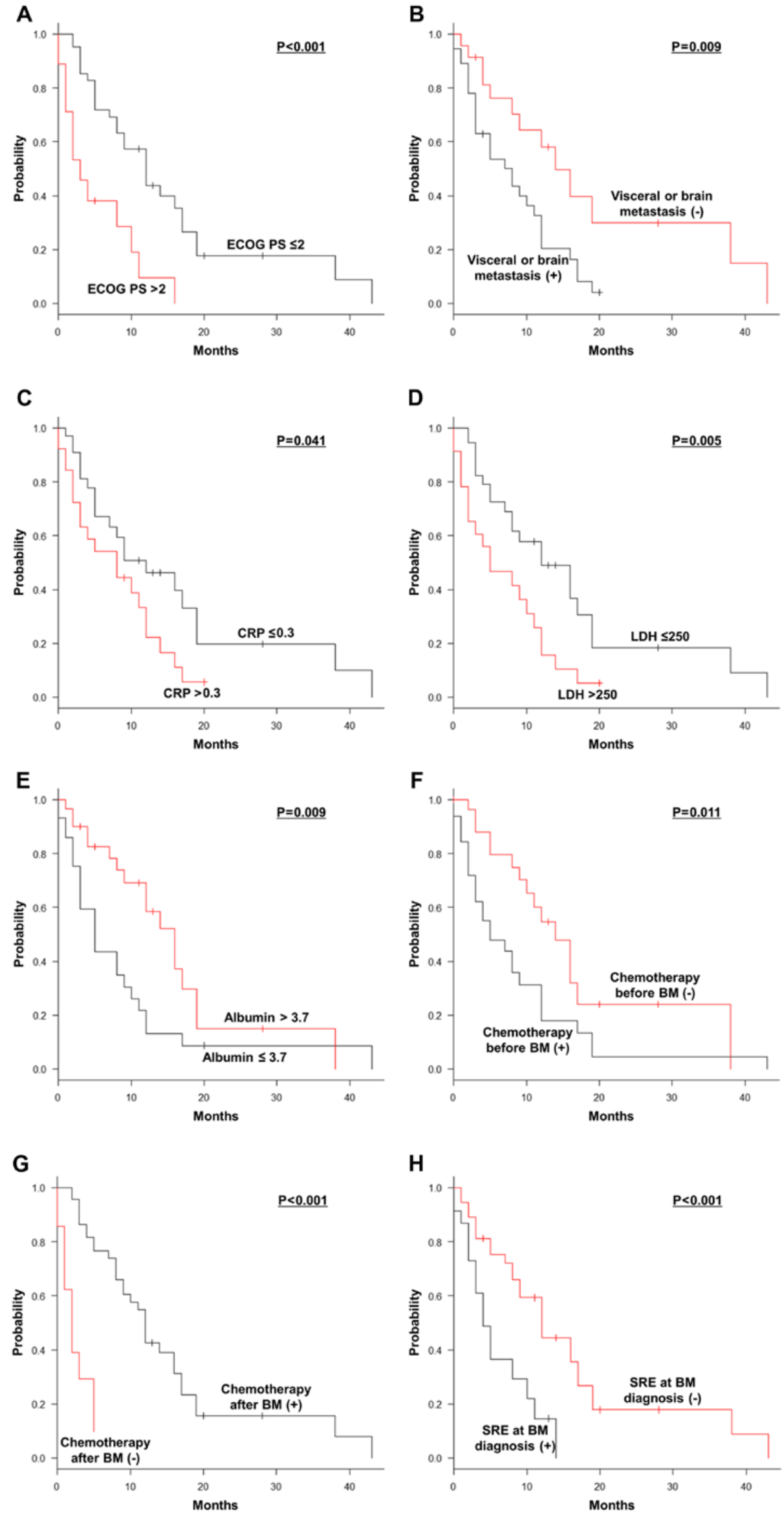

Figure 1. Kaplan-Meier survival curve for OS and univariate analysis for significant prognostic factors. (A) ECOG PS score; (B) visceral or brain metastasis; (C) serum CRP level; (D) serum LDH level; (E) serum albumin level; (F) chemotherapy before the diagnosis of BM. (G) Chemotherapy after the diagnosis of BM. (H) SREs at diagnosis of BM. OS, overall survival; ECOG PS, Eastern Cooperative Oncology Group performance status; CRP, C-reactive protein; LDH, lactate dehydrogenase; BM, bone metastasis; SRE, skeletal-related event. 
Table II. Multivariate analysis of prognostic factors for OS.

\begin{tabular}{lllr}
\hline Factors & HR & 95\% CI & P-value \\
\hline ECOG PS score $>2$ & 3.165 & $1.120-8.945$ & 0.030 \\
Visceral or brain metastasis & 1.965 & $0.950-4.065$ & 0.069 \\
Chemotherapy before BM diagnosis & 3.802 & $1.812-7.976$ & $<0.001$ \\
No chemotherapy after BM diagnosis & 5.897 & $1.926-18.050$ & 0.002 \\
SRE at BM diagnosis & 2.000 & $0.655-6.112$ & 0.224
\end{tabular}

OS, overall survival; HR, hazard ratio; CI, confidence interval; ECOG PS, Eastern Cooperative Oncology Group performance status; BM, bone metastasis; SRE, skeletal-related event.

Table III. Patient-, tumor- and BM-related characteristics and univariate analysis of prognostic factors for SRS $(n=37)$.

\begin{tabular}{|c|c|c|}
\hline Factors & $\mathrm{N}(\%)$ & $\begin{array}{r}\text { Median SI } \\
\text { (months }\end{array}$ \\
\hline \multicolumn{3}{|c|}{ Age (years) } \\
\hline$\leq 60$ & $20(54.1)$ & 4.5 \\
\hline$>60$ & $17(45.9)$ & 16 \\
\hline \multicolumn{3}{|c|}{ ECOG PS score } \\
\hline $0-2$ & $34(91.9)$ & 5 \\
\hline $3-4$ & $3(8.1)$ & 16 \\
\hline \multicolumn{3}{|c|}{ Resection of primary site } \\
\hline Present & $17(45.9)$ & 7 \\
\hline Absent & $20(54.1)$ & 8.5 \\
\hline \multicolumn{3}{|c|}{$\begin{array}{l}\text { Visceral or brain } \\
\text { metastasis }\end{array}$} \\
\hline Present & $23(62.2)$ & 5 \\
\hline Absent & $14(37.8)$ & 15 \\
\hline \multicolumn{3}{|c|}{ CEA (ng/ml) } \\
\hline$\leq 5$ & $12(33.3)$ & 12 \\
\hline$>5$ & $24(66.7)$ & 5 \\
\hline N/A & 1 & \\
\hline \multicolumn{3}{|c|}{ CA19-9 (U/ml) } \\
\hline$\leq 37$ & $15(40.5)$ & 12 \\
\hline$>37$ & $22(59.5)$ & 4 \\
\hline \multicolumn{3}{|c|}{ CRP (mg/dl) } \\
\hline$\leq 0.3$ & $26(72.2)$ & 7 \\
\hline$>0.3$ & $10(27.8)$ & 5 \\
\hline $\mathrm{N} / \mathrm{A}$ & 1 & \\
\hline \multicolumn{3}{|c|}{ LDH (U/l) } \\
\hline$\leq 250$ & $26(70.3)$ & 5 \\
\hline$>250$ & $11(29.7)$ & 10 \\
\hline \multicolumn{3}{|c|}{ Albumin (g/dl) } \\
\hline$\leq 3.7$ & $15(41.7)$ & 4 \\
\hline$>3.7$ & $21(58.3)$ & 10 \\
\hline N/A & 1 & \\
\hline \multicolumn{3}{|c|}{$\operatorname{ALP}(\mathrm{U} / \mathrm{l})$} \\
\hline$\leq 350$ & $18(48.6)$ & 10 \\
\hline$>350$ & $19(51.4)$ & 4 \\
\hline \multicolumn{3}{|c|}{$\mathrm{BM}$ at $\mathrm{GC}$ diagnosis } \\
\hline Present & $8(21.6)$ & 16 \\
\hline Absent & $29(78.4)$ & 4 \\
\hline
\end{tabular}

0.582

0.903

0.486
Table III. Continued.

\begin{tabular}{lccc}
\hline Factors & N (\%) & $\begin{array}{c}\text { Median SRS } \\
\text { (months) }\end{array}$ & P-value \\
\hline Number of BM & $8(21.6)$ & 7.5 & 0.800 \\
$\quad$ Solitary & $29(78.4)$ & 7 & \\
$\quad$ Multiple & & & 0.103 \\
Chemotherapy before & & & \\
BM diagnosis & $23(62.2)$ & 4 & \\
$\quad$ Present & $14(37.8)$ & 12 & 0.970 \\
Absent & & & \\
Chemotherapy after & & & \\
BM diagnosis & $34(91.9)$ & 7 & 0.008 \\
Present & $3(8.1)$ & 5 & \\
Absent & & & \\
Use of BMA & $22(59.5)$ & 12 & \\
Present & $15(40.5)$ & 3 & \\
Absent &
\end{tabular}

BM, bone metastasis; SRS, skeletal-related event-free survival; ECOG PS, Eastern Cooperative Oncology Group performance status; CEA, carcinoembryonic antigen; CA19-9, carbohydrate antigen 19-9;

0.107 CRP, C-reactive protein; LDH, lactate dehydrogenase; ALP, alkaline phosphatase; GC, gastric cancer; BMA, bone-modifying agent.

Table IV. Multivariate analysis of prognostic factors for SRS.

\begin{tabular}{lccc}
\hline Factors & HR & $95 \%$ CI & P-value \\
\hline No BM at GC diagnosis & 2.699 & $0.863-8.439$ & 0.088 \\
Non-use of BMA & 2.868 & $1.163-7.076$ & 0.022
\end{tabular}

0.593 SRS, skeletal-related event-free survival; HR, hazard ratio; CI, confidence interval; BM, bone metastasis; BMA, bone-modifying agent; N/A, not available. visceral or brain metastasis $(61.7 \%)$, were of the osteolytic
type $(40 \%)$, and occurred most commonly in the spine $(81.7 \%)$ and pelvis $(61.7 \%)$. Moreover, the serum ALP level, known to be the most predictive biological marker for the presence of $\mathrm{BM}$ in $\mathrm{GC}(9,16)$, was elevated in $63.3 \%$ of the patients in 
A

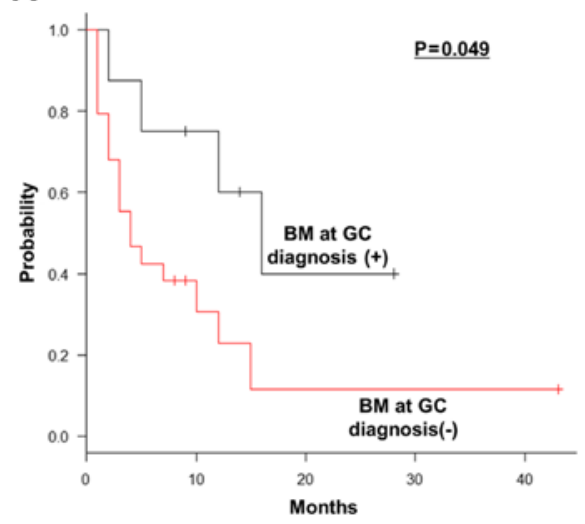

B

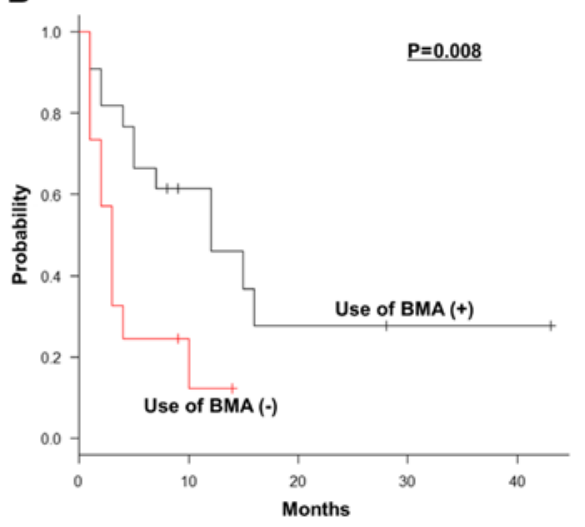

Figure 2. Kaplan-Meier survival curve for SRS and univariate analysis for significant prognostic factors. (A) BM at the diagnosis of GC; (B) use of BMA. SRS, skeletal-related event-free survival; BM, bone metastasis; GC, gastric cancer; BMA, bone-modifying agent.

the present study. In general, liver metastasis, in addition to BM, may be associated with elevated levels of ALP. However, although $\gamma$-glutamyl transpeptidase $(\gamma$-GTP) activity correlates with cholestatic liver disease, $\gamma$-GTP activity is not usually increased in BM. In the present study, $\gamma$-GTP levels were not increased in 18 of the $21(85.7 \%)$ patients with high serum levels of ALP who did not have liver metastasis (data not shown). Therefore, it is suggested that, in patients diagnosed with GC, if the ALP value is atypically elevated, measurement of $\gamma$-GTP should be included in the evaluation of BM. Even if GC patients are asymptomatic, elevated serum levels of ALP and tumor markers, such as CEA and CA19-9, indicate BM and evaluation of $\mathrm{BM}$ is required. In the present study, $43.3 \%$ of the patients were asymptomatic at diagnosis of BM, and either bone scintigraphy or FDG-PET/CT was performed in such patients. However, as the serum ALP level is not always elevated when BM is present, using appropriate modalities, such as CT, MRI, bone scintigraphy and FDG-PET/CT, may also be necessary in routine practice, even in asymptomatic patients, in order to detect BM at an early stage.

Patients with metastatic GC with good ECOG PS scores and organ function should be offered systemic chemotherapy for palliation in order to improve survival. The most frequently used standard first-line chemotherapy regimen for metastatic GC is a combination of a fluoropyrimidine with platinum, although triple regimens including docetaxel may be useful in otherwise healthy patients with a high tumor burden $(17,18)$. In patients with good ECOG PS score and organ function, second-line treatment with agents not used in the first-line treatments, such as taxanes and irinotecan, may confer a modest survival benefit $(19,20)$. By contrast, chemotherapy is rarely administered to patients with poor ECOG PS. Previous studies have reported poor prognosis of BM from GC, with a median OS of 2-7 months after BM diagnosis (3-7,14,21). However, the median OS period after BM diagnosis in the present study was 9 months, indicating that the prognosis of GC patients with BM in our study population was better compared with that previously reported in the literature. BM in $\mathrm{GC}$ is often associated with a rapidly deteriorating clinical course and extremely poor prognosis, due to combined bone marrow metastasis causing hematological abnormalities, such as disseminated intravascular coagulation (DIC). In the present study, $3.3 \%$ patients who had DIC at diagnosis of BM succumbed to the disease within 2 months after the diagnosis of BM and $21.7 \%$ of the patients developed DIC during the clinical course (data not shown). Poor ECOG PS score was one of the worst prognostic factors for OS on multivariate analyses. Moreover, significantly poor OS was also found on multivariate analyses in GC patients receiving chemotherapy prior to BM diagnosis and without palliative chemotherapy after BM occurrence. These data suggest that further treatment options may be available for GC patients who have not received chemotherapy compared with those with intensive chemotherapy prior to BM diagnosis; furthermore, even if BM has been diagnosed, regardless of the presence of visceral or brain metastasis, palliative chemotherapy after BM diagnosis should be considered whenever possible.

Currently, palliative radiotherapy for BM associated with pain symptoms is a well-established treatment. Previous studies have reported that BM causes high rates of SREs in patients with GC and radiotherapy is the most common SRE (3-6). Concordantly, our data also revealed that SREs occurred in $76.7 \%$ of our patients, and that radiotherapy for BM was performed in the majority $(73.3 \%)$ of those patients. In the present study, the median survival after SRE occurrence was only 5 months, possibly due to aggressive SREs affecting survival, or other complications associated with SREs. Approximately $50 \%$ of patients who experienced SREs were found to have had these SREs at BM diagnosis, which was a significant prognostic factor for poor OS on univariate analysis. The data of the present study demonstrated that, among patients who did not present with SREs at BM diagnosis, 91.9\% had ECOG PS scores 0-2 and were considered as candidates for palliative chemotherapy after BM diagnosis. These data suggest that early detection of BM before the occurrence of SRE improves patient survival.

It has been established that BMA, such as zoledronic acid and denosumab, are beneficial for the treatment and prevention of skeletal complications in patients with multiple myeloma and breast and prostate cancers (22-24). However, prospective data on the efficacy of BM in GC are lacking in the literature. In the present study, although this treatment modality did not significantly prolong OS, it was associated with a significant extension of SRS. Therefore, our data support the beneficial 
effects of BMA for BM that occurs from GC. At present, preventive dental care to decrease the risk of developing medication-related osteonecrosis of the jaw is usually offered before administering BMAs in our institution, and BMAs are generally well tolerated. Even if asymptomatic, the initiation of BMA treatment upon diagnosis of BM is recommended to delay time to SREs and reduce skeletal morbidity in GC patients with BM.

There were certain limitations to the present study. This retrospective study was performed without randomization of patient selection. The number of patients was insufficient to draw a definitive conclusion. The majority of BM cases were not confirmed pathologically. The standardized methods used for detecting BMs were heterogenous, with each methodology having its own limit of detection. We were unable to obtain tumor stage data or laboratory test data in some of the cases. The present study did not quantify and compare the therapeutic effects of chemotherapy due to the wide range of chemotherapy regimens utilized. Finally, the usage of BMAs depended on the discretion of the attending physician.

In conclusion, poor ECOG PS score often prevents patients from receiving further available treatment. Therefore, early detection of BM and optimal treatment with BMA is imperative for preventing or delaying SREs, leading to maintenance of a more favorable ECOG PS score and continuation of chemotherapy in patients with GC.

\section{Acknowledgements}

Not applicable.

\section{Funding}

The present study was supported by the Japan Orthopedics and Traumatology Research Foundation, Inc. (grant no. 372) and JSPS KAKENHI (grant no. JP19K18481).

\section{Availability of data and materials}

The datasets used and analyzed during the present study are available from the corresponding author on reasonable request.

\section{Authors' contributions}

YI and DT designed the study. DT, NS, AI, TW, HO, TTan, HT, TY, NN and ST collected, interpreted and analyzed the data. YI, DT, SO, TTab and ST performed statistical analyses. YI wrote the manuscript. NS and TY treated the patients. DT, NS, AI, TW, HO, TTan, HT, TY, NN, SO, TTab and ST revised the manuscript critically for important intellectual content. All the authors have read and approved the final manuscript.

\section{Ethics approval and consent to participate}

The study protocol was approved by the Institutional Review Board of the Osaka International Cancer Institute (Osaka, Japan).

\section{Patient consent for publication}

Not applicable.

\section{Competing interests}

All the authors declare that they have no competing interests.

\section{References}

1. Jemal A, Bray F, Center MM, Ferlay J, Ward E and Forman D: Global cancer statistics. CA Cancer J Clin 61: 69-90, 2011.

2. Guadagni S, Catarci M, Kinoshita T, Valenti M, De Bernardinis G and Carboni M: Causes of death and recurrence after surgery for early gastric cancer. World J Surg 21: 434-439, 1997.

3. Ahn JB, Ha TK and Kwon SJ: Bone metastasis in gastric cancer patients. J Gastric Cancer 11: 38-45, 2011.

4. Park HS, Rha SY, Kim HS, Hyung WJ, Park JS, Chung HC, Noh SH and Jeung HC: A prognostic model to predict clinical outcome in gastric cancer patients with bone metastasis. Oncology 80: 142-150, 2011.

5. Silvestris N, Pantano F, Ibrahim T, Gamucci T, De Vita F, Di Palma T, Pedrazzoli P, Barni S, Bernardo A, Febbraro A, et al: Natural history of malignant bone disease in gastric cancer: Final results of a multicenter bone metastasis survey. PLoS One 8: e74402, 2013.

6. Turkoz FP, Solak M, Kilickap S, Ulas A, Esbah O, Oksuzoglu B and Yalcin S: Bone metastasis from gastric cancer: The incidence, clinicopathological features, and influence on survival. J Gastric Cancer 14: 164-172, 2014.

7. Kim YJ, Kim SH, Kim JW, Lee JO, Kim JH, Bang SM, Lee JS and Lee KW: Gastric cancer with initial bone metastasis: A distinct group of diseases with poor prognosis. Eur J Cancer 50: 2810-2821, 2014

8. Nishidoi H and Koga S: Clinicopathological study of gastric cancer with bone metastasis. Gan To Kagaku Ryoho 14: 1717-1722, 1987 (In Japanese).

9. Choi CW, Lee DS, Chung JK, Lee MC, Kim NK, Choi KW and Koh CS: Evaluation of bone metastases by Tc-99m MDP imaging in patients with stomach cancer. Clin Nucl Med 20: 310-314, 1995.

10. Nielsen OS, Munro AJ and Tannock IF: Bone metastases: Pathophysiology and management policy. J Clin Oncol 9: 509-524, 1991.

11. Mundy GR: Metastasis to bone: Causes, consequences and therapeutic opportunities. Nat Rev Cancer 2: 584-593, 2002.

12. Coleman RE: Clinical features of metastatic bone disease and risk of skeletal morbidity. Clin Cancer Res 12 (Suppl): 6243S-6249S, 2006.

13. Berenson JR, Rosen LS, Howell A, Porter L, Coleman RE, Morley W, Dreicer R, Kuross SA, Lipton A and Seaman JJ: Zoledronic acid reduces skeletal-related events in patients with osteolytic metastases. Cancer 91: 1191-1200, 2001.

14. Riihimaki M, Hemminki A, Sundquist K, Sundquist J and Hemminki K: Metastatic spread in patients with gastric cancer. Oncotarget 7: 52307-52316, 2016.

15. Coleman RE: Metastatic bone disease: Clinical features, pathophysiology and treatment strategies. Cancer Treat Rev 27: 165-176, 2001.

16. Lim SM, Kim YN, Park KH, Kang B, Chon HJ, Kim C, Kim JH and Rha SY: Bone alkaline phosphatase as a surrogate marker of bone metastasis in gastric cancer patients. BMC Cancer 16: 385, 2016.

17. Van Cutsem E, Moiseyenko VM, Tjulandin S, Majlis A, Constenla M, Boni C, Rodrigues A, Fodor M, Chao Y, Voznyi E, et al: Phase III study of docetaxel and cisplatin plus fluorouracil compared with cisplatin and fluorouracil as first-line therapy for advanced gastric cancer: A report of the V325 Study Group. J Clin Oncol 24: 4991-4997, 2006.

18. Cunningham D, Starling N, Rao S, Iveson T, Nicolson M, Coxon F, Middleton G, Daniel F, Oates J and Norman AR: Capecitabine and oxaliplatin for advanced esophagogastric cancer. N Engl J Med 358: 36-46, 2008.

19. Kang JH, Lee SI, Lim DH, Park KW, Oh SY, Kwon HC, Hwang IG, Lee SC, Nam E, Shin DB, et al: Salvage chemotherapy for pretreated gastric cancer: A randomized phase III trial comparing chemotherapy plus best supportive care with best supportive care alone. J Clin Oncol 30: 1513-1518, 2012.

20. Ford HE, Marshall A, Bridgewater JA, Janowitz T, Coxon FY, Wadsley J, Mansoor W, Fyfe D, Madhusudan S, Middleton GW, et al: Docetaxel versus active symptom control for refractory oesophagogastric adenocarcinoma (COUGAR-02): An open-label, phase 3 randomised controlled trial. Lancet Oncol 15: 78-86, 2014. 
21. Mikami J, Kimura Y, Makari Y, Fujita J, Kishimoto T, Sawada G, Nakahira S, Nakata K, Tsujie M and Ohzato H: Clinical outcomes and prognostic factors for gastric cancer patients with bone metastasis. World J Surg Oncol 15: 8, 2017.

22. Rosen LS, Gordon D, Kaminski M, Howell A, Belch A, Mackey J, Apffelstaedt J, Hussein MA, Coleman RE, Reitsma DJ, et al: Long-term efficacy and safety of zoledronic acid compared with pamidronate disodium in the treatment of skeletal complications in patients with advanced multiple myeloma or breast carcinoma: A randomized, double-blind, multicenter, comparative trial Cancer 98: 1735-1744, 2003.

23. Fizazi K, Carducci M, Smith M, Damiao R, Brown J, Karsh L, Milecki P, Shore N, Rader M, Wang H, et al: Denosumab versus zoledronic acid for treatment of bone metastases in men with castration-resistant prostate cancer: A randomised, double-blind study. Lancet 377: 813-822, 2011.
24. Stopeck AT,Lipton A, Body JJ, Steger GG, Tonkin K, de Boer RH Lichinitser M, Fujiwara Y, Yardley DA, Viniegra M, et al: Denosumab compared with zoledronic acid for the treatment of bone metastases in patients with advanced breast cancer: A randomized, double-blind study. J Clin Oncol 28: 5132-5139, 2010.

This work is licensed under a Creative Commons Attribution-NonCommercial-NoDerivatives 4.0 International (CC BY-NC-ND 4.0) License. 